\title{
SMO classification for cervical cancer dataset by applying various kernels
}

\author{
Dr.G.Ayyappan \\ K.SivaKumar \\ SIPS Technologies, \\ Chennai, Tamil Nadu, India.
}

Assistant Professor, Department of Information Technology, Bharath University

\begin{abstract}
This research work presents a decision making of healthcare operational system by using machine learning classifiers algorithm to predict the decision making in comparison to the actual decision making. This model may help to doctor for making the best decisions. This model helps us to prediction of indicators/diagnosis of cervical cancer. This study explains utilization of machine learning algorithms in determination of medical operation methods. This dataset focuses on the prediction of indicators/diagnosis of cervical cancer. The results show that SMO in RBF Kernel parameter for this case study generates highest accuracy level of $87.5 \%$.
\end{abstract}

\section{INTRODUCTION}

Machine learning in today's healthcare is unavoidable. Optimists predict that machine learning and artificial intelligence will diagnose disease better and earlier, treat illness more precisely and engage patients more efficiently in future healthcare. In recent years instances in healthcare such as medical image processing and analyzing, predicting healthcare operational decisions, dosage trials for intravenous tumor treatment detection and management of prostate cancer.In this paper organizes section one has related works and brief introduction of this fields, section two presents Materials and Methods, the section three describes results and discussions and the section four presents conclusion.

\section{MATERIALS AND METHODS}

In this section presents the materials and methods of this research work. Here the dataset borrowed from https://archive.ics.uci.edu/ml/datasets/Cervical+cancer+\%28Risk+Factors\%29\# . In this dataset contains information about The dataset comprises demographic information, habits, and historic medical records of 858 patients. In this research work applied in weka 3.8.3 version for SMO classification method by applying various kernels namely Polykernel, Normalized Polykernel, Puk, and RBF Kernel were applied to calculate for predicting caesarian section operational decisions

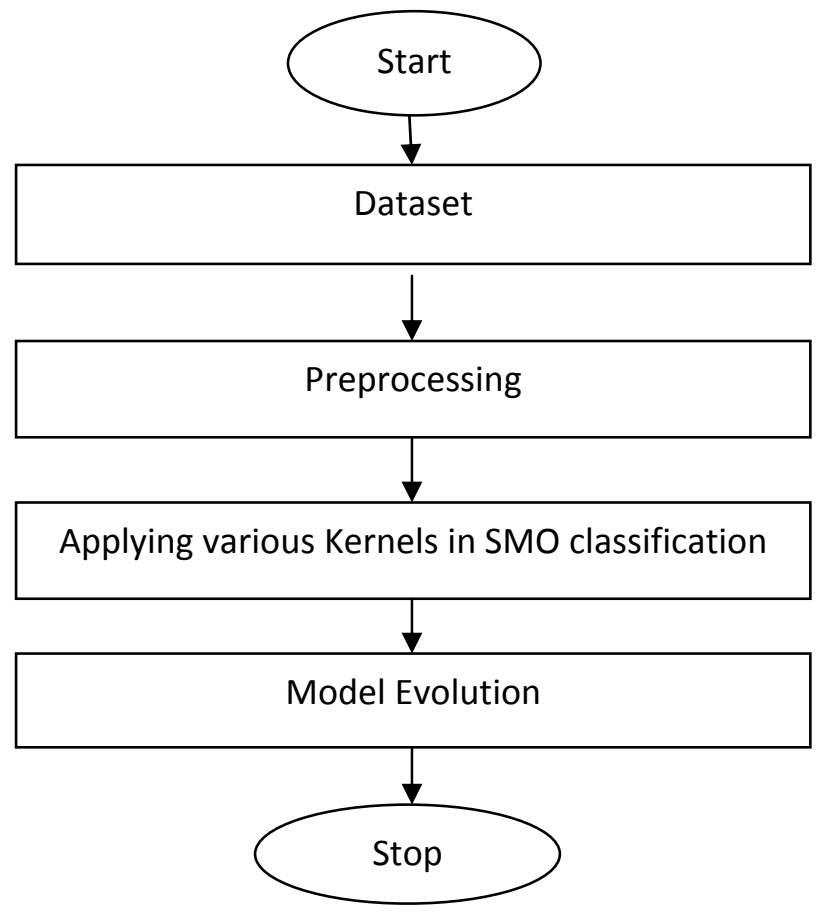

Figure 1 Architecture of Proposed method 


\section{RESULTS AND DISCUSSIONS}

In this section presents results and interpretations of this research work. Machine learning needs of the healthcare organization regarding operational decision making.

Table 1 Accuracy level of SMO Classification by using different kernels

\begin{tabular}{|c|c|c|}
\hline S.No & Kernels in SMO & Accuracy level \\
\hline 1 & Polykernel & $75.54 \%$ \\
\hline 2 & Normalized Polykernel & $68.43 \%$ \\
\hline 3 & Puk & $72.33 \%$ \\
\hline 4 & RBF Kernel & $87.5 \%$ \\
\hline
\end{tabular}

In this SMO machine learning algorithm by applying various kernels namely Polykernel, Normalized Polykernel, Puk, and RBF Kernel were applied to calculate for predicting caesarian section operational decisions.

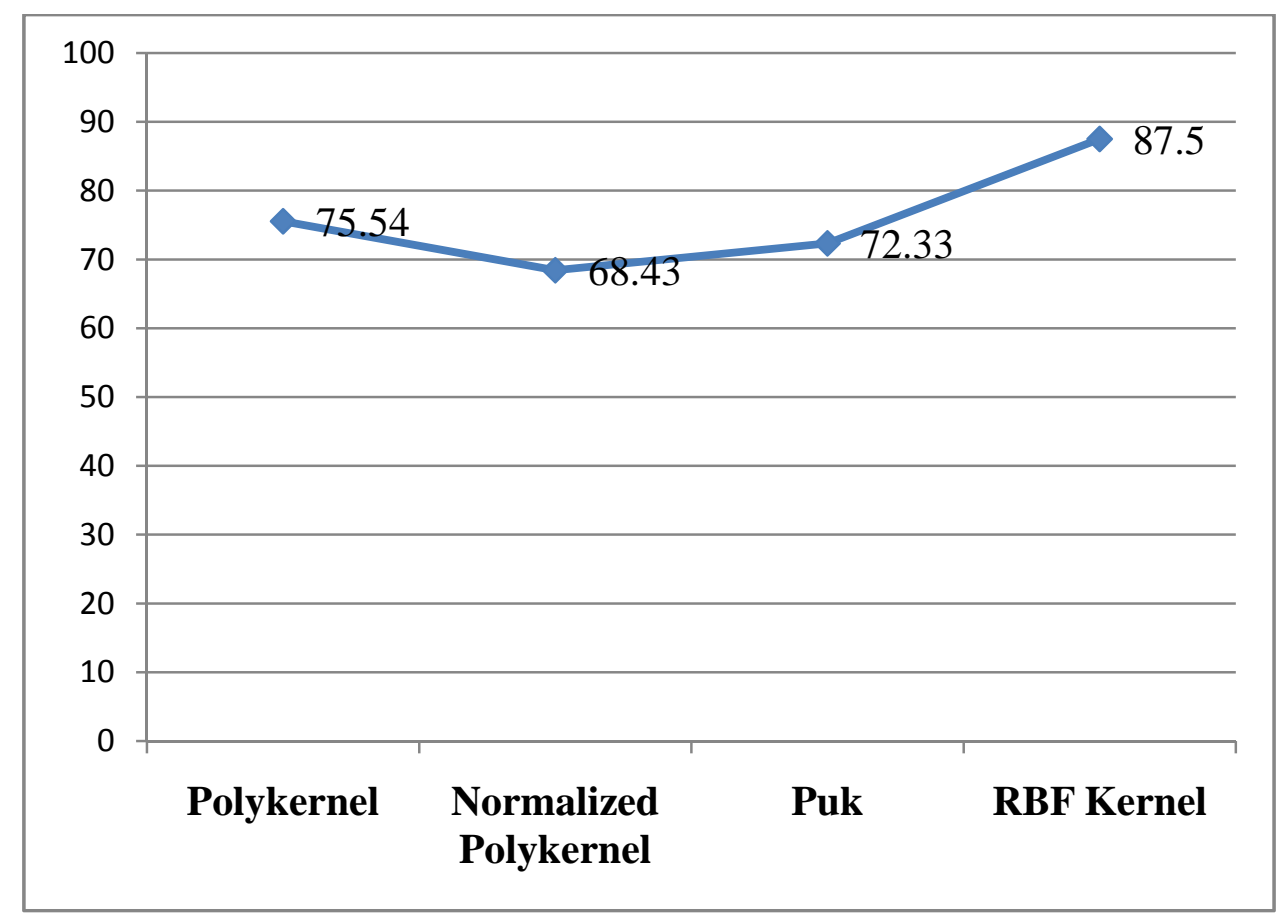

Figure 2 Accuracy level of SMO Classification by using different kernels

The above diagram clearly demonstrates Polykernel has $75.54 \%$ accuracy level, Normalized Polykernel has 68.43\%accuracy level, Puk has 72.33\% accuracy level, and RBF Kernel has 87.5\% accuracy level.

\section{CONCLUSION}

The experiment results show that SMO with RBF Kernel has 87.5\% accuracy level achieved the best accuracy rates by using the dataset comprises demographic information, habits, and historic medical records of 858 patients.So, this research work recommended for decision making based on the SMO with RBF Kernel classification.

\section{REFERENCES}

[1] (PDF) Performance Evaluation of Supervised Machine Learning Classifiers for Predicting Healthcare Operational Decisions. Available from: https://www.researchgate.net/publication/329515048_Performance_Evaluation_of_Supervised_Machine_Learning_Classifiers_for_Pr edicting_Healthcare_Operational_Decisions [accessed Jan 06 2019].

[2] Boris Milovic, MilanMilovic. Prediction and Decision Making in Health Care using Data Mining, International Journal of Public Health Science (IJPHS), Vol. 1, No. 2, December 2012, pp. 69 78

[3] Fayyad, U., Shapiro, G. P., \& Smyth, P. (1996). From Data Mining to Knowledge Discovery in Databases. American Association for Artificial Intelligence, 37-54.

[4] H. Jiawei and K. Micheline, Data Mining: Concepts and Techniques, vol. 2, Morgan Kaufmann Publishers, 2006.

[5] Candelieri, A., Dolce, G., Riganello, F., \&Sannita, W. G. (2011). Data Mining in Neurology. In KnowledgeOriented Applications in Data Mining (pp. 261-276). InTech. 
[6] Brady E. Hamilton, Ph.D.; Joyce A. Martin, M.P.H.; and Stephanie J. Ventura, M.A., Division of Vital Statistics, Births: Preliminary Data for 2007, National Vital Statistics Report. [7] Births: Provisional Data for 2017 USA. CDC. May 2018. Retrieved 18 May 2018.

[7] World Health Organization (WHO) statement "Should there be a limit on Caesareans?". BBC News. 30 June 2010.

[8] Andrew Simm, Darly Mathew, Caesarian section: techniques and complications, Obstetrics, Gynaecology\& Reproductive Medicine, Volume 18, Issue 4, April 2008, Pages 93-98.

[9] FarhadSoleimanianGharehchopogh, PeymanMohammadi, ParvinHakimi, Application of Decision Tree Algorithm for Data Mining in Healthcare Operations: A Case Study, International Journal of Computer Applications (0975 - 8887) Volume 52 - No. 6, August 2012,Pages 21-26. 